\title{
Iron Deficiency Alters Iron Regulatory Protein and Iron Transport Protein Expression in the Perinatal Rat Brain
}

\author{
ASHA JYOTHI M. SIDDAPPA, RAGHAVENDRA B. RAO, JANE D. WOBKEN, \\ KELLY CASPERSON, ELIZABETH A. LEIBOLD, JAMES R. CONNOR, AND \\ MICHAEL K. GEORGIEFF
}

\begin{abstract}
Division of Neonatology, Department of Pediatrics [A.J.M.S, R.B.R., J.D.W., K.C., M.K.G.], Center for Neurobehavioral Development [R.B.R., M.K.G.], University of Minnesota Medical School, Minneapolis, MN 55455, U.S.A.; Department of Medicine, Eccles Program in Human Molecular Biology and Genetics [E.A.L], University of Utah, Salt Lake City, UT 84112, U.S.A.; and Department of Neuroscience and Anatomy [J.R.C.], Pennsylvania State University College of Medicine, Hershey, PA 17033, U.S.A.
\end{abstract}

\begin{abstract}
Iron plays an important role in numerous vital enzyme systems in the perinatal brain. The membrane proteins that mediate iron transport [transferrin receptor (TfR) and divalent metal transporter 1 (DMT-1)] and the iron regulatory proteins (IRP-1 and IRP-2) that stabilize their mRNAs undergo regional developmental changes in the iron-sufficient rat brain between postnatal day (P) 5 and 15. Perinatal iron deficiency (ID) affects developing brain regions nonhomogeneously, suggesting potential differences in regional iron transporter and regulatory protein expression. The objective of the study was to determine the effect of perinatal ID on regional expression of IRP-1, IRP-2, TfR, and DMT-1 in the developing rat brain. Gestationally iron-deficient Sprague Dawley rat pups were compared with iron-sufficient control pups at P10. Serial $12-\mu$ coronal sections of fixed frozen brain from pups on P10 were assessed by light microscopy for IRP-1, IRP-2, DMT-1, and TfR localization. ID did not change the percentage of cells with positive staining for the four proteins in the choroid epithelium, ependyma, vascular endothelium, or neurons of the striatum. ID increased the percentage of neurons
\end{abstract}

\section{ABSTRACT}

expressing the four proteins in the hippocampus and the cerebral cortex. Increased numbers of TfR- and DMT-1-positive cells were always associated with increased IRP-positive cells. The P10 rat responds to perinatal ID by selectively increasing the number of neurons expressing IRP-regulated transporters in brain regions that are rapidly developing, without any change at transport surfaces or in regions that are quiescent. Brain iron distribution during ID seems to be locally rather than globally regulated. (Pediatr Res 53: 800-807, 2003)
ID, iron deficiency
TfR, transferrin receptor
DMT-1, divalent metal transporter 1
IRP, iron regulatory protein
CytOx, cytochrome $c$ oxidase
IRE, iron responsive element
$\mathbf{P}$, postnatal day

Iron is essential for normal CNS growth and development because it forms an important component of hemoproteins and enzymes involved in neuronal oxidative metabolism, neurotransmitter synthesis, and myelin synthesis (1). Iron deficiency (ID) during late fetal and early postnatal life in humans $(2,3)$ and in animal models is associated with cognitive impairments that persist despite iron repletion (4). These deficits are pre-

Received July 19, 2002; accepted December 18, 2002.

Correspondence: Michael K. Georgieff, M.D., University of Minnesota, Mayo Mail Code 39, 420 Delaware Street SE, Minneapolis, MN 55455, U.S.A.; e-mail: georg001@umn.edu

Supported by NIH grants HD-29421 (MKG), GM-45201 (E.A.L.), NS 34280 (J.R.C.), and HD39386 (M.K.G. and J.R.C.).

DOI: 10.1203/01.PDR.0000058922.67035.D5 sumably due to alterations in iron-based neuronal cellular processes that occur during a period of rapid brain growth and its associated high iron demand (5).

ID during the perinatal period also results in regionalization of functional brain iron (6-8). Regionalization suggests that the brain has a mechanism for sensing iron requirements and for prioritizing iron to high-need areas. We have previously shown that a $40 \%$ decrease in total brain iron content in the perinatal rat results in differential regional losses of cytochrome $c$ oxidase (CytOx), an iron-containing enzyme, with more marked deficits noted in the hippocampus and prefrontal cortex than in the striatum on postnatal day (P) 10 (6). The preferential loss of hippocampal CytOx in our model is consistent with deficits in the neural circuits underlying recogni- 
tion and working memory demonstrated by Felt and Lozoff (4) in a similar model.

The mechanism by which the perinatal brain regionalizes iron distribution during deficiency states is not understood $(6-8)$. The effect may be dependent on the relative responsiveness of the iron transporters transferrin receptor (TfR) and divalent metal transporter-1 (DMT-1) and their putative regulatory proteins iron regulatory proteins (IRP) 1 and 2 to ID. The mature brain accretes iron across two surfaces, the bloodbrain barrier and the blood-CSF-brain interface $(9,10)$. Regulated iron transport across these surfaces and subsequent iron uptake by glia and neurons are dependent on TfR and DMT-1. The expression of TfR and possibly DMT-1 is regulated posttranscriptionally by two cytosolic iron regulatory proteins, IRP1 and IRP2 $(11,12)$. IRP are sequence- and structurespecific RNA binding proteins found in the cytosol. They are sensitive to decreased intracellular iron status and bind ironresponsive elements (IRE) on the 3' untranslated regions of TfR and DMT-1 mRNA when intracellular iron is low. The binding increases the stability of the mRNA and increases the expression of the respective uptake proteins, resulting in restoration of intracellular iron status (11-13). Whereas IRP-1's sensitivity to iron status results in a conformational change that enhances IRE binding, IRP-2 increases its chances of binding IRE through increased protein expression (13). The locations of IRP expressing cells in the brain have been mapped in the iron-sufficient developing rat (14) and in the adult mouse (15). The distribution of cells expressing the IRP is different in the adult and developing rodent. The effect of iron deficiency on the location and types of cells expressing the two IRP during iron deficiency has not been previously studied.

All four proteins undergo marked increases in regional expression in the iron-sufficient rat between P5 and P15, particularly in areas of high metabolic activity (e.g. hippocampus, cortex). This developmental change is characterized by increases in the percentage of neuronal and nonneuronal cells expressing these proteins (14).

We hypothesized that a $40 \%$ reduction in brain iron would increase the number of cells expressing TfR, DMT-1, and IRP at the blood-brain transport surfaces, including the vascular endothelium (blood-brain barrier), choroid plexus epithelium (blood-CSF barrier), and ependymal endothelium (CSF-brain barrier). Furthermore, our previous study on CytOx loss suggested that perinatal ID induces large differences in regional iron status. We therefore hypothesized that a given degree of ID would increase the number of cells expressing the iron transporters and their regulators in brain regions with the highest CytOx loss such as the hippocampus and cerebral cortex, whereas areas with minimal CytOx loss such as the striatum would show little change.

\section{METHODS}

\section{Animal Preparation and Study Design}

The experiment was approved by the Institutional Animal Care and Utilization Committee of the University of Minnesota. Six iron-sufficient and six iron-deficient Sprague Dawley rat pups were studied on P10. Dams of iron-sufficient pups were maintained on a standard iron-fortified (198 mg/kg food) diet (4\% mouse/rat diet, Teklad, Madison, WI, U.S.A.) throughout pregnancy and postpartum, whereas dams of irondeficient pups were maintained on a low-iron $(3-6 \mathrm{mg} / \mathrm{kg}$ food) diet (Formula TD 80396, Harlan Teklad) from gestational day 2 until P10. This diet results in a $45 \%$ loss of total brain iron concentration in the pups by P10 (16).

The pups were weighed and were deeply anesthetized with sodium pentobarbital $(60 \mathrm{mg} / \mathrm{kg}$ i.p.). Blood samples were obtained for determination of hematocrit, and the pups were transcardially perfused with $0.9 \% \mathrm{NaCl}$. The brains were rapidly removed and postfixed in a solution of $5 \%$ formaldehyde and 5\% sucrose in PBS ( $\mathrm{pH} 7.4$ ) for $24 \mathrm{~h}$ at $4^{\circ} \mathrm{C}$. They were cryoprotected by serial passages through $20 \%, 30 \%$, and $40 \%$ sucrose in PBS over a period of several days before being quick-frozen in a tissue-embedding medium (Fischer Scientific, Fair Lawn, NJ, U.S.A.). Serial $12-\mu$ coronal sections were obtained using a cryostat (Model CM1900; Leica Instruments $\mathrm{GmbH}$, Nussloch, Germany) at $-25^{\circ} \mathrm{C}$ to $-27^{\circ} \mathrm{C}$. The brain sections were mounted on poly-L-lysine-coated slides and were stored at $-80^{\circ} \mathrm{C}$ until immunohistochemical analyses were performed. On the basis of our previous studies $(6,14)$, brain sections that included the hippocampus, the prefrontal cortex, and the striatum were selected for analysis. The choroid plexus was assessed in the third ventricle, and the ependymal cell layer was assessed in the linings of the third and fourth ventricles.

\section{Immunohistochemistry}

Immunohistochemical analysis for each protein was performed simultaneously in iron-deficient and iron-sufficient animals using multiple sections. As with our previous study (14), primary antibodies were diluted in $0.01 \mathrm{M}$ PBS (pH 7.4) with $2 \%$ Triton $\mathrm{X}-100$ and $2 \%$ horse serum as follows: the primary chicken anti-rat IRP-1 antibody (17), 1:250, the rabbit anti-rat IRP-2 antibody (14), 1:750, the rabbit anti-DMT-1 antibody (18), 1:300, and the mouse anti-rat TfR antibody (Research Diagnostics, Flanders, NJ, U.S.A.), 1:400. The two IRP antibodies have been previously shown to recognize IRP in developing rat (14) and adult mouse (15) brains. The DMT-1 antibody recognizes both the IRE and non-IRE form of the molecule in the developing and adult rat brain $(14,18)$. The brain sections were pretreated in a solution of $1 \%$ horse serum for IRP-2, DMT-1, and TfR and in 1\% goat serum for IRP-1 for $20 \mathrm{~min}$ before incubation with the respective primary antibody. They were treated with avidin D followed by biotin (Avidin/Biotin Blocking Kit, Vector Laboratory, Burlingame, CA, U.S.A.) (15 min each, with a rinse with PBS for $5 \mathrm{~min}$ between treatments) to block all endogenous avidin and biotin binding sites before adding biotinylated secondary antibodies (14).

Brain sections were rinsed in PBS and were incubated with their respective primary antibody ( $2 \mathrm{~h}$ each for IRP-1, IRP-2, and DMT-1 and overnight for TfR) (14). After incubation, they were treated for $30 \mathrm{~min}$ in a biotinylated secondary corresponding to the species of the primary antibody, followed by streptavidin/peroxidase complex for $30 \mathrm{~min}$ (Vector Laboratory). 
They were incubated with 3, 3'-diaminobenzidine (Sigma Chemical Co., St. Louis, MO, U.S.A.) for $5 \mathrm{~min}$ to reveal the bound peroxidase. After rinses in water to stop the reaction, brain sections were then dehydrated in a series of increasing concentrations of ethyl alcohol, cleared in Histoclear (Fisher Scientific), and coverslipped with a permanent mounting medium.

\section{Microscopic Visualization and Analysis}

General principles. Brain sections were visualized under a light microscope (Nikon Optiphot, Nippon Kogaku K.K., Tokyo, Japan) at 10-1000 $\times$ magnification. The anatomic regions of interest were identified as in our previous studies and were assessed from their most anterior to their most posterior aspect in the P10 brain $(6,14,19)$. Anatomic structures were sampled in a minimum of three and a maximum of seven planes with each brain sampled at least in the most anterior and most posterior aspects. In the hippocampus, cortex, and striatum, a $10 \times 10$-mm grid inserted in the eyepiece was used to define the extent of the region of interest during counting at $400 \times$ and to ensure accurate cell counts. The type of cell assessed varied on the basis of the region of interest. Sample cell counts were first performed on control (no primary antibody) slides to determine the expected cell numbers for each region without introducing any visual bias of stained versus unstained cells. Subsequently, the number of cells of specific interest with positive staining for IRP-1, IRP-2, DMT-1, and TfR was expressed as percentage of all of the cells (positive and negative) of that type in the region. The results across the six animals in each group were averaged, and the mean percentage was categorized into $<25 \%$ positive cells, $25-75 \%$ positive cells, and $>75 \%$ positive cells. The percentage of positively stained cells from the iron-sufficient and iron-deficient brains was compared.

Specific brain regions. The brain regions of interest were the potential transport surfaces including the choroid plexus, ependymal lining and blood vessels, the hippocampus, the cerebral cortex, and the head of the caudate nucleus (striatum). The cell types of interest were the cuboidal epithelial cells of the choroid, the ependymal cells of the ventricles, the endothelial cells of the vasculature, the pyramidal neurons of the cortex and hippocampal subareas CA1 and CA3, the granular cells of the lower blade of the dentate gyrus, and the neurons of the head of the caudate.

Staining of the choroid plexus cells of both lateral ventricles was assessed from 0 to $5.0 \mathrm{~mm}$ anterior to the interauricular line. The choroid plexus of the third ventricle was assessed between 0 and $3.4 \mathrm{~mm}$ anterior to the same reference point (19). All choroid epithelial cells in a given section were counted, although the choroid was frequently damaged and thus incomplete on sections. The epithelial cell count for each choroid plexus was approximately 600 cells, and at least four choroids were assessed per animal. Staining of the central blood vessels in the choroid was not included in the counts.

Staining of ependymal cells was assessed in the third and fourth ventricles from 0 to $3.4 \mathrm{~mm}$ anterior to the interauricular line. The ependyma of both ventricles was typically intact and averaged 350 cells per ventricle. All ependymal cells were counted, and the number of positively stained cells was expressed as a percentage of the total number of cells.

The three hippocampal subareas (CA1, CA3c, and dentate gyrus) were assessed separately because of the different functions subserved by these areas and because the dentate has predominantly granular cells whereas the CA areas are populated by pyramidal cells. Only these primary neuronal cell types were assessed for positive staining. The hippocampus was assessed from 0.0 to $2.6 \mathrm{~mm}$ anterior to the interauricular line (19). All pyramidal cells in CA1 were counted from its medial border to $2 \mathrm{~mm}$ laterally (approximately 500 cells). All pyramidal cells in CA3c were counted in a 1-mm area defined by the posterolateral corner of the structure (approximately 300 cells). All granular cells in the lower blade of the dentate gyrus were counted for $1 \mathrm{~mm}$ laterally from its medial border.

The pyramidal cells of the developing cortical layers were counted from the interauricular line to a point $6.5 \mathrm{~mm}$ anteriorly using the grid (19). At $400 \times$ magnification, 18 nonoverlapping grid areas could be assessed in the section at the interauricular line. The cortical cell counts ranged from 2000 to 3600 cells, depending on whether the counting was anterior or posterior. The cortex was further subdivided into inner, middle, and outer layers of approximately 1000 cells each.

The neurons of the head of the caudate nucleus (striatum) were counted at $400 \times$ magnification from 4.4 to $6.5 \mathrm{~mm}$ anterior to the interauricular line using the grid (19). Twelve nonoverlapping grid areas were assessed per section. Neuron counts ranged from 500 to 700 .

\section{RESULTS}

The model induced significant ID in the experimental pups. The mean \pm SD hematocrit was $38 \pm 3 \%$ in the iron-sufficient pups and $19 \pm 4 \%$ in the iron-deficient pups $(p<0.001)$. The staining patterns of IRP-1, IRP-2, and DMT-1 were characterized by a granular pattern throughout the cytoplasm and the neuronal axonal processes. Neuronal TfR staining was found on the cell membrane surface. The findings in the ironsufficient group were consistent with previous experiments at P10 (14).

Choroid plexus epithelium, ependyma, and vascular endothelium. Less than $25 \%$ of choroid plexus epithelial cells stained positively for the four proteins. The percentage of stained choroid cells was similar between the lateral and third ventricles, and the percentage did not change from anterior to posterior. Iron deficiency did not increase the percentage of stained cells (Table 1). One hundred percent of ependymal cells stained positively for IRP-1, IRP-2, and DMT-1 in both the iron-sufficient and iron-deficient brains. Less than $10 \%$ of ependymal cells stained positively for TfR in either condition. The percentages did not change in the rostral-caudal plane (Table 1). The iron-sufficient vascular endothelium stained positively for all four proteins in both conditions. Figure 1 demonstrates the lack of effect of ID on TfR staining in choroid and ependymal cells.

Hippocampus. The effects of ID on staining for the four proteins in the three hippocampal subareas are shown in Table 
Table 1. Effect of ID on the percentage of positively stained cells in the choroid epithelium, the ependymal cell layer, and the vascular endothelium

\begin{tabular}{lcc}
\hline Transport surface/protein & Iron sufficient & Iron deficient \\
\hline Choroid plexus epithelium & & \\
IRP-1 & + & + \\
IRP-2 & + & + \\
DMT-1 & ++ & ++ \\
TfR & $0 /+$ & $0 /+$ \\
Ependyma & & \\
IRP-1 & +++ & +++ \\
IRP-2 & +++ & +++ \\
DMT-1 & +++ & +++ \\
TfR & $0 /+$ & $0 /+$ \\
Vascular endothelium & & \\
IRP-1 & +++ & +++ \\
IRP-2 & +++ & +++ \\
DMT-1 & +++ & +++ \\
TfR & +++ & +++ \\
\hline
\end{tabular}

0 , no positively stained cells;,$+<25 \%$ positively stained cells; ++ $25-75 \%$ positively stained cells;,$+++ 75-100 \%$ positively stained cells.

2. In the iron-sufficient brain, the percentage of positively stained pyramidal cells in CA1 and CA3c was greater than the percentage of positively stained granular cells in the dentate gyrus. ID increased the number of stained neurons for IRP-1, IRP-2, DMT-1, and TfR in all hippocampal subareas. The dentate gyrus showed the greatest change in percentage of cells stained for all four proteins in response to ID. The results were similar in rostral and caudal sections for all four proteins in all three subareas under both dietary conditions. Figure 2 demonstrates the change in the effect of ID on IRP-1 and DMT-1 staining in the granular cells of the dentate gyrus.

Cerebral cortex. The effects of ID on the percentage of positively stained pyramidal neurons for the four proteins in the cerebral cortex are shown in Table 3. The percentages were unaffected by anterior versus posterior location. In the ironsufficient brain, cortical neuronal staining for all four proteins was expressed predominantly in the mid-cortical layers, with some positive neurons noted in the outer layer. In contrast, in the deficient state, all layers of the cerebral cortex had a high percentage of neurons stained positively for all four proteins. Figure 3 demonstrates the increase in staining for IRP-1 and DMT-1 in all cortical layers in the iron-deficient animal.

Striatum. Neurons were positively stained for IRP-1, IRP-2, DMT-1, and TfR in the iron-sufficient striatum, and the percentage of cells that were positive did not change with ID. Figure 4 demonstrates the lack of differences between ironsufficient and iron-deficient animals in staining for IRP-2 in the striatum.

\section{DISCUSSION}

Newborn infants of mothers with diabetes mellitus and infants with evidence of intrauterine growth retardation have high rates of fetal and neonatal ID. Greater than $50 \%$ of these infants are born with abnormally low iron stores (20-22), and $25 \%$ are estimated to be at risk for brain ID (21). Autopsy studies in these infants have shown a $30-40 \%$ decrease in brain iron content $(21,23)$. Electrophysiologic studies have shown abnormalities in hippocampally based recognition memory processing in newborn infants of mothers with diabetes, suggesting iron-dependent impairments of neurodevelopment (24). Follow-up studies of these infants demonstrate continued poorer long-term cognitive processing despite iron repletion (25).

In the perinatal rat brain, iron deficiency similar to that seen in human infants results in depletion of ferritin iron throughout the brain but widely variable losses of the functional iron compound CytOx (6). The greater loss of CytOx activity in subareas of the hippocampus and the cortex compared with the minimal loss in the striatum, amygdala, and entorhinal area suggests that the neurons in the former areas have a greater degree of intracellular ID (6). Thus, we hypothesized in the current study that IRP-1, IRP-2, DMT-1, and TfR protein expression (as indexed by the number of cells with positive

\section{IS}

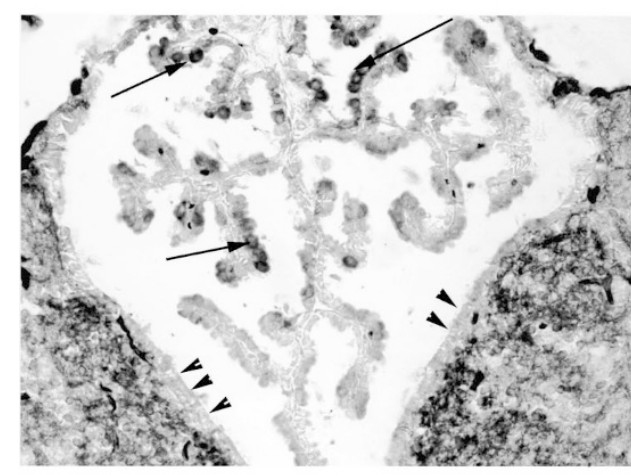

ID

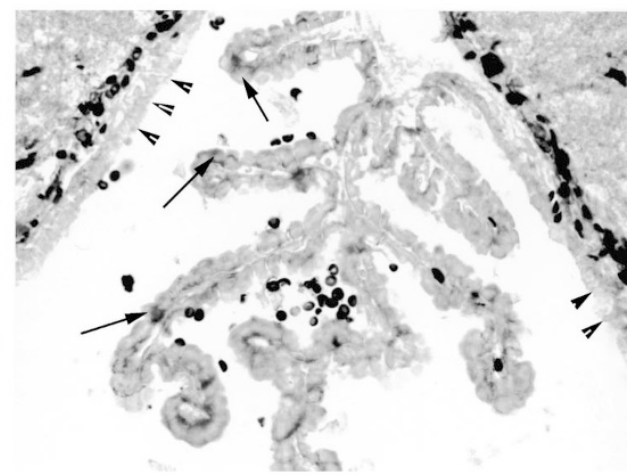

Figure 1. Choroid plexus epithelial cells and ependymal cells lining the third ventricle from iron-sufficient (left) and iron-deficient (right) brain stained for TfR. Absent staining in the ependymal cells is marked with arrowheads. Examples of positively stained choroid epithelial cells are marked with arrows. Positively stained red cells and blood vessels are visible in both sections. Magnification: $\times 400$. 
Table 2. Effect of ID on IRP-1, IRP-2, DMT-1, and TfR staining of pyramidal neurons in subareas $C A 1$ and $C A 3 c$ of the hippocampus and granular cells of the dentate gyrus

\begin{tabular}{lcc}
\hline Hippocampal subarea/protein & Iron sufficient & Iron deficient \\
\hline CA1 & & \\
IRP-1 & ++ & +++ \\
IRP-2 & ++ & +++ \\
DMT-1 & ++ & +++ \\
TfR & + & +++ \\
CA3 & & \\
IRP-1 & ++ & +++ \\
IRP-2 & ++ & +++ \\
DMT-1 & ++ & +++ \\
TfR & + & +++ \\
Dentate gyrus & & \\
IRP-1 & $0 /+$ & +++ \\
IRP-2 & $0 /+$ & +++ \\
DMT-1 & $0 /+$ & +++ \\
TfR & $0 /+$ & ++ \\
\hline
\end{tabular}

0 , no positively stained cells;,$+<25 \%$ positively stained cells; ++ , $25-75 \%$ positively stained cells;,$+++ 75-100 \%$ positively stained cells.

staining for these proteins) would be increased in these irondeficient regions as the cells attempted to regain intracellular iron sufficiency. The results of the current study supported this hypothesis. We found increased numbers of neurons with detectable IRP-1, IRP-2, DMT-1, and TfR in the brain subregions that were previously shown to be most deficient in CytOx activity. We found no increases in the area where CytOx activity was spared (6).

The brain has two potential mechanisms to increase iron delivery to iron-starved neurons. The more general mechanism would be to increase total brain iron delivery from the serum via the blood-brain and the blood-CSF-brain interfaces (10,
26). This mechanism seems to be the normal response of the more mature animal where brain iron uptake increases 2-fold in response to dietary iron deficiency (26). This process would be dependent on the vascular endothelial cell of the blood-brain barrier, the choroid plexus epithelial cell of the blood-CSF barrier, and the ependymal cell of the CSF-brain barrier being able to sense the total iron status of the brain. In the immature animal with incomplete expression of the transport proteins (14), the expected response to ID would be to increase the percentage of cells expressing the proteins or to increase the amount of protein (e.g. staining intensity) within the cells. All cells in the ependyma and the vascular endothelium had positive staining in the iron-sufficient state, and thus no increase could be appreciated with ID. However, we saw no evidence for iron deficiency increasing the numbers of stained cells in the choroid epithelium, which modulates blood-CSF iron transport. A more specific mechanism to increase uptake would be based on the intracellular iron concentration of individual neuronal and nonneuronal cells in a given region. This mechanism allows for some degree of control on the basis of regional iron supply and neuronal demand and is likely present in the more mature animal $(7,8)$. We found evidence for this mechanism in the perinatal animal, as well, as judged by the increased number of cells expressing the proteins in the hippocampus and the cortex and the contrast between the findings in those areas and the striatum.

The effects of ID on neuronal iron transporters and IRP expression in the three brain regions varied by anatomic subregion. We chose to study the hippocampus, the cerebral cortex, and the striatum for three reasons. First, these areas represented the extremes of the spectrum of ID-induced CytOx
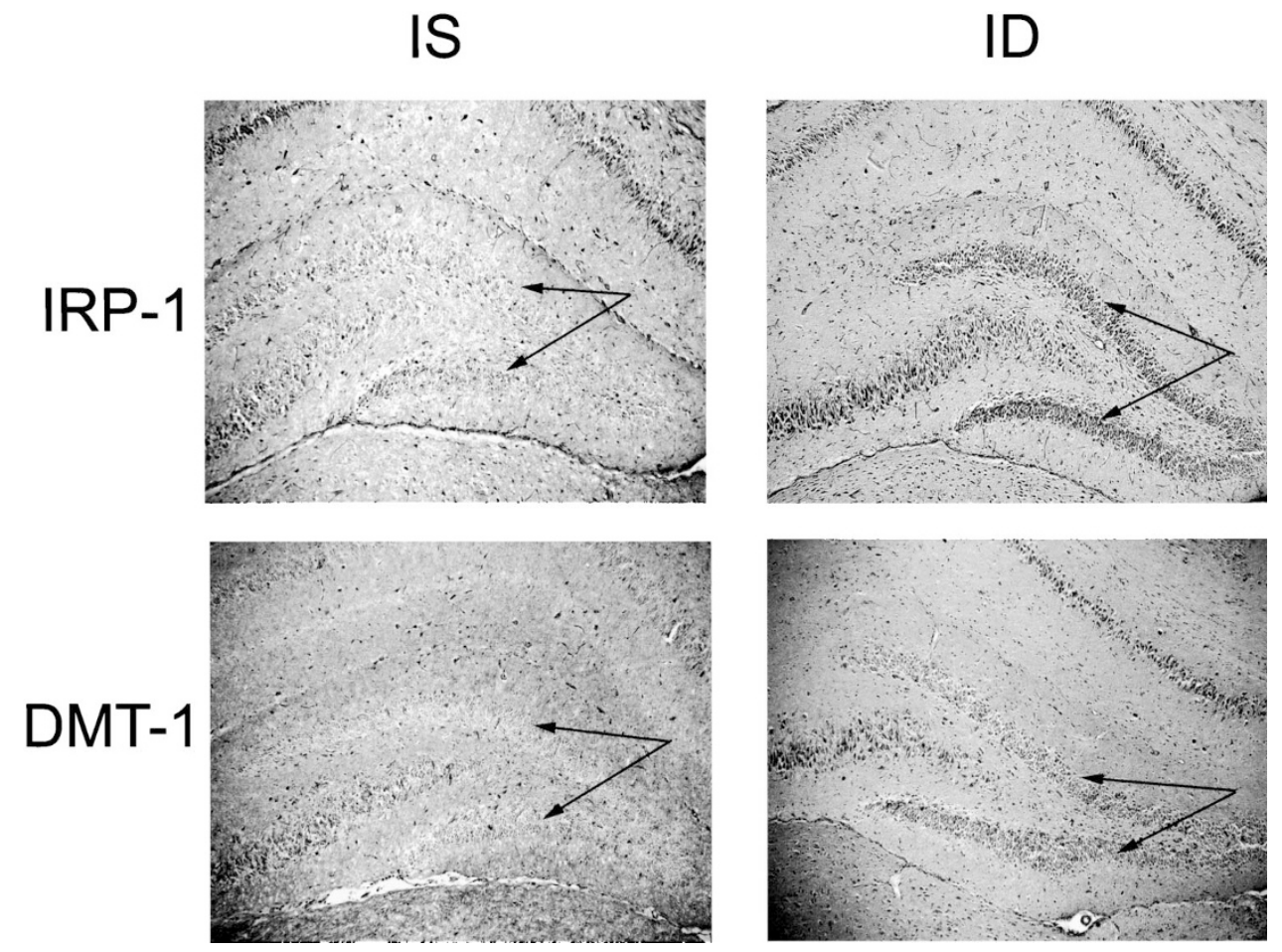

Figure 2. IRP-1 (top) and DMT-1 (bottom) staining in iron-sufficient (left) and iron-deficient (right) brain in the upper and lower blades of the dentate gyrus (arrows). Note the marked increase in the number of dentate granular cells expressing both proteins in the iron-deficient brain. Magnification: $\times 10$. 
Table 3. Effect of ID on the percentage of pyramidal neurons with positive staining for the IRP, DMT-1, and TfR in the cerebral cortex

\begin{tabular}{lcc}
\hline Cortical layer/protein & Iron sufficient & Iron deficient \\
\hline Outer layer & & + \\
IRP-1 & ++ & ++ \\
IRP-2 & ++ & +++ \\
DMT-1 & + & +++ \\
TfR & & \\
Middle layers & ++ & +++ \\
IRP-1 & ++ & +++ \\
IRP-2 & ++ & +++ \\
DMT-1 & ++ & +++ \\
TfR & & +++ \\
Inner layers & + & +++ \\
IRP-1 & + & +++ \\
IRP-2 & + & +++ \\
DMT-1 & + & + \\
TfR & + & + \\
\hline
\end{tabular}

0 , no positively stained cells;,$+<25 \%$ positively stained cells; ++ $25-75 \%$ positively stained cells;,$+++ 75-100 \%$ positively stained cells.

activity loss at P10, with the hippocampus and cortex exhibiting the greatest loss and the striatum the least (6). Second, at P10, the cerebral cortex and the hippocampus become metabolically more active and would be expected to have a high iron requirement, whereas the striatum is metabolically relatively quiescent (27). Third, these brain regions subserve the two major memory systems: explicit (recognition) and implicit (procedural) memory (28). The hippocampus with its CA1, $\mathrm{CA} 3 \mathrm{c}$, and dentate gyrus subregions involved in long-term potentiation and learning is at the core of recognition memory processing (28). Lesions of the hippocampus result in profound impairments of recognition memory, spatial memory, and spatial working memory $(29,30)$. Downstream effects via connec- tions to the prefrontal cortex include altered executive functions. Iron-deficient rats perform abnormally on the Morris water maze task (4), which is thought to rely on intact recognition and spatial working memory systems. The striatum, particularly the head of the caudate, is central to procedural learning and seems to function independent of the hippocampus (28).

In the hippocampus, ID increased the numbers of cells and cell types that stained positively for IRP-1, IRP-2, DMT-1, and TfR. The dentate gyrus normally has minimal expression of these proteins until P15 in the iron-sufficient rat (14). ID at P10 induced $>75 \%$ of the dentate granular cells to express the proteins, effectively making the area seem developmentally 5 days older. The CA areas of the hippocampus already express low levels of the proteins at P10 in the iron-sufficient state (14). The marked increase in the number of cells with positive staining suggests that these areas that are involved in long-term potentiation have significant ongoing ID. Previous studies have reported that cortex and hippocampal areas are sensitive to ID and lose iron rapidly (7). Assessing the status of the transporters and their IRP may be a useful way to demonstrate that these cells are iron deficient.

In the cerebral cortex, ID induced the cortical neurons in the middle layers to increase the number of cells with positive staining for proteins already present at P10 in the ironsufficient state. It also altered the time of onset of protein expression in the inner and outer cortical layers-areas that would not normally be expressing the proteins on P10 (14). The iron-deficient cortex at $\mathrm{P} 10$ had the staining pattern of an iron-sufficient cortex at P15, with all layers expressing both IRP and transporters (14). These findings suggest that the

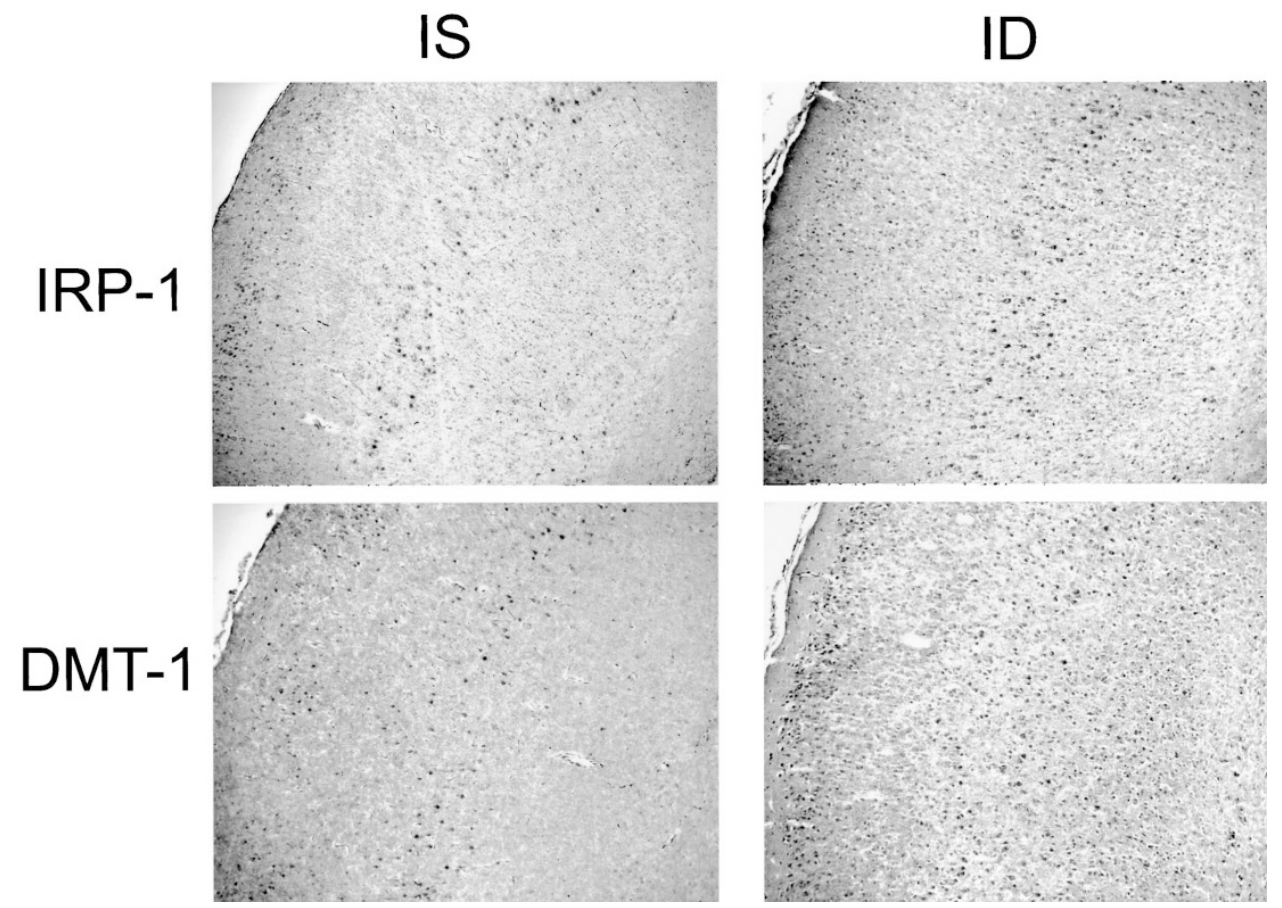

Figure 3. IRP-1 (top) and DMT-1 (bottom) staining in iron-sufficient (left) and iron-deficient (right) cerebral cortex. In contrast to the iron-sufficient brain, neurons in all layers are stained in the iron-deficient brain. Magnification: $\times 100$. 


\section{IS}

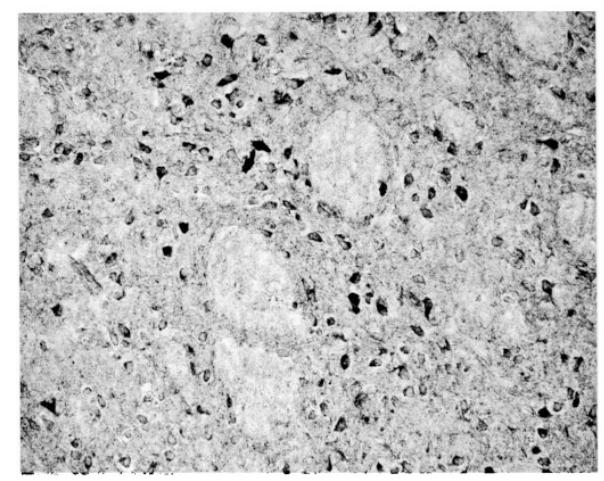

ID

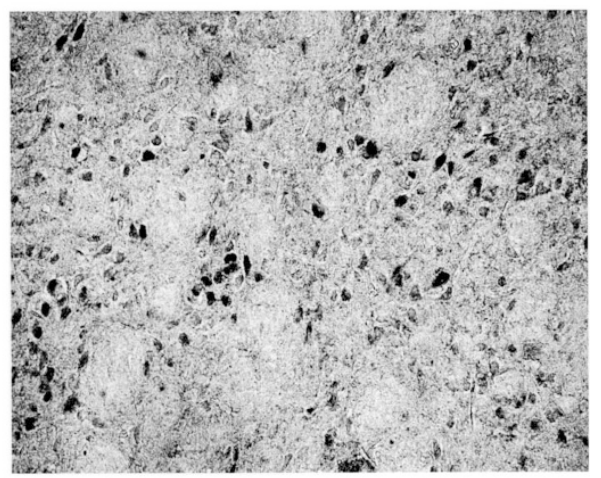

\section{IRP-2}

Figure 4. IRP-2 staining of striatum in an iron-sufficient (left) and an iron-deficient (right) brain. Magnification: $\times 400$.

cortex is profoundly iron deficient at a time when its metabolic demands are increasing.

In contrast, the number of cells that stained positive for the four proteins in the striatum was similar in the iron-sufficient and iron-deficient state. The findings are consistent with this area remaining relatively sufficient in functional iron despite the $40 \%$ decrease in total brain iron induced by this model (6). The mechanism(s) for the preservation of iron status in this area is not known but may be related to a relatively low iron demand during this period of development. We speculate that at P10, the striatum is already mature compared with the hippocampus and the cerebral cortex, and therefore its metabolic demands are low. Thus, a decrease in supply seems to have little effect on intracellular iron status and consequently on IRP and iron transporter status. Extending these speculations to brain function, it seems that striatum-based behaviors might be less affected by perinatal ID than hippocampus-based behaviors.

ID in the perinatal animal presents unique challenges to the brain because it occurs at a time when there is a systematic developmental ontogeny of these proteins (14). The apparent disruption and hastening of the expected developmental changes in brain iron uptake as seen in the dentate gyrus could potentially affect long-term brain development by disrupting the delicate timing involved in iron delivery for rapidly dividing and differentiating neurons and myelin-producing oligodendrocytes.

Immunohistochemistry proved to be a useful method for studying the effect of ID on the developing brain because it allowed us to assess localization (e.g. TfR on the neuronal cell membrane) as well as the number of cells with detectable protein. Immunohistochemistry is not a strictly quantitative technique in that the increases in staining intensity within cells may or may not reflect increases in protein quantity. Nevertheless, the striking increase in the number of cells in which there was detectable staining strongly supports the premise that ID has activated the iron trans- porters in selective regions. Assessment of the IRP and particularly IRP-2 gave us meaningful insight in transporter mRNA regulation $(13,31)$. IRP-2 seems to be a strong regulator of TfR mRNA because degradation of IRP-2 in cell culture results in a rapid decrease TfR mRNA levels (31). In our study, we speculate that cells that stain positively for the IRP-2 have more stable TfR mRNA and thus more copies of the proteins than cells that do not stain positively. Thus, the number of cells expressing detectable levels of IRP-2 can be used as a functional assay of the degree of iron deficiency in a given brain region. In this respect, the increase in the number of cells that stained positively for IRP-2 in the hippocampus of ID animals confirms the high degree of ID in that particular region.

Although ID seems to activate this regulated transporter system, function as defined by increased iron uptake was not assessed in this study. mRNA can be stabilized, more transporter protein can be made, and more cells can express the transporters, but kinetic studies would be needed to demonstrate more active iron transport in the ID regions. Nevertheless, with the iron transporters studied in cell culture systems, presence of the protein typically correlates with increased iron transport. Similarly, if IRP-2 is not seen, the cell is either iron sufficient and IRP-2 has been degraded or the cell does not express that particular IRP $(13,15)$.

\section{CONCLUSION}

In summary, our results show that the rat brain at P10 responds to perinatal ID by selectively increasing the percentage of neurons with detectable iron regulatory and transport proteins in a regional manner. The effect is greater in the hippocampus and cortex compared with the striatum, suggesting that the first two are functionally more iron deficient than the last in this model. ID during this rapid period of brain maturation has the potential to alter neuroanatomy and neurometabolism in these areas, resulting in long-term neuronal dysfunction and behavioral impairment. 
Acknowledgments. We thank Jennifer Welle for technical help in the laboratory and Ginny Lyson for editorial assistance in the manuscript preparation.

\section{REFERENCES}

1. Dallman PR 1986 Biochemical basis for the manifestations of iron deficiency. Annu Rev Nutr 6:13-40

2. Lozoff B, Jimenez E, Hagen J, Mollen E, Wolf AW 2000 Poorer behavioral and developmental outcome more than 10 years after treatment for iron deficiency in infancy. Pediatrics 105(4). Available at: www.pediatrics.org/cgi/content/full/105/4/

3. Walter T, De Andraca I, Chadud P, Perales CG 1989 Iron deficiency anemia: adverse effects on infant psychomotor development. Pediatrics 84:7-17

4. Felt BT, Lozoff B 1996 Brain iron and behavior of rats are not normalized by treatment of iron deficiency anemia during early development. J Nutr 126:693-701

5. Lozoff B 2000 Perinatal iron deficiency and the developing brain. Pediatr Res 48:137-139

6. deUngria M, Rao R, Wobken JD, Luciana M, Nelson CA, Georgieff MK 2000 Perinatal iron deficiency decreases cytochrome c oxidase activity in selected regions of neonatal rat brain. Pediatr Res 48:169-176

7. Erikson KM, Pinero DJ, Connor JR, Beard JL 1997 Regional brain iron, ferritin and transferrin concentrations during iron deficiency and iron repletion in developing rats. J Nutr 127:2030-2038

8. Pinero DJ, Lin NQ, Connor JR, Beard JL 2000 Variations in dietary iron alter brain iron metabolism in developing rats. J Nutr 130:254-263

9. Moos T, Morgan EH 2000 Transferrin and transferrin receptor function in brain barrier systems. Cell Mol Neurobiol 20:77-95

10. Burdo JR, Connor JR 2001 Iron transport in the central nervous system. In: Templeton D (ed) Cellular and Molecular Mechanism of Iron Transport. Marcel Dekker Inc., New York, pp 487-505

11. Hentze MW, Kuhn LC 1996 Molecular control of vertebrate iron metabolism: mRNA-based regulatory circuits operated by iron, nitric oxide, and oxidative stress. Proc Natl Acad Sci U S A 93:8175-8182

12. Gunshin H, Allerson CR, Polycarpou-Schwarz M, Rofts A, Rogers JT, Kishi F, Hentze MW, Roualt TA, Andrews NC, Hediger MA 2001 Iron dependent regulation of the divalent metal ion transporter. FEBS Lett 509:309-316

13. Eisenstein RS 2000 Iron regulatory proteins and the molecular control of mammalian iron metabolism. Annu Rev Nutr 20:627-662

14. Siddappa AJM, Rao R, Wobken JD, Leibold EA, Connor JR, Georgieff MK 2002 Developmental changes in the expression of iron regulatory proteins and iron transport proteins in the perinatal iron brain. J Neurosci Res 68:761-775
15. Leibold EA, Gehring LC, Rogers SW 2000 Immunolocalization of iron regulatory protein expression in the murine central nervous system. Histochem Cell Biol 115:195-203

16. Rao R, de Ungria M, Sullivan D, Wu P, Wobken JD, Nelson CA, Georgieff MK 1999 Perinatal brain iron deficiency increases the vulnerability of rat hippocampus to hypoxic ischemic insult. J Nutr 129:199-206

17. Guo B, Yu Y, Leibold EA 1994 Iron regulates cytoplasmic levels of a novel iron-responsive element-binding protein without aconitase activity. J Biol Chem 269:24252-24260

18. Burdo JR, Menzies SL, Simpson IA, Garrick LM, Garrick MD, Dolan KG, Haile DJ, Beard JL, Connor JR 2001 Distribution of divalent metal transporter 1 and metal transport protein 1 in normal and Belgrade rat. J Neurosci Res 66:1198-1207

19. Sherwood NM, Timiras PS 1970 A Stereotaxic Atlas of the Developing Rat Brain. University of California Press, Berkeley, CA

20. Chockalingam UM, Murphy E, Ophoven JC, Weisdorf SA, Georgieff MK 1987 Cord transferrin and ferritin values in newborn infants at risk for prenatal uteroplacental insufficiency and chronic hypoxia. J Pediatr 111:283-286

21. Georgieff MK, Landon MB, Mills MM, Hedlund BE, Faassen AE, Schmidt RL, Ophoven JJ, Widness JA 1990 Abnormal iron distribution in infants of diabetic mothers: spectrum and maternal antecedents. J Pediatr 117:455-461

22. Petry CD, Wobken JD, McKay H, Eaton MA, Seybold VS, Johnson DE, Georgieff MK 1994 Placental transferrin receptor in diabetic pregnancies with increased fetal iron demand. Am J Physiol 267:E507-E514

23. Georgieff MK, Petry CD, Wobken JD, Oyer CE 1996 Liver and brain iron deficiency in newborn infants with bilateral renal agenesis (Potter's syndrome). Pediatr Pathol Lab Med 16:509-519

24. deRegnier RA, Nelson CA, Thomas K, Wewerka S, Georgieff MK 2000 Neurophysiologic evaluation of auditory recognition memory in healthy newborn infants and infants of diabetic mothers. J Pediatr 137:777-784

25. Nelson CA, Wewerka S, Thomas KM, Tribby-Walbridge S, deRegnier R, Georgieff M 2000 Neurocognitive sequelae of infants of diabetic mothers. Behav Neurosci 114:950-956

26. Taylor EM, Crowe A, Morgan EH 1991 Transferrin and iron uptake by the brain: effects of altered iron status. J Neurochem 57:1584-1592

27. Rice D, Barone S 2000 Critical periods of vulnerability for the developing nervous system: evidence from human and animal models. Environ Health Perspect 108:511533

28. Nelson CA 1995 The ontogeny of human memory. Dev Psychol 31:723-738

29. Zola-Morgan S, Squire LR 1986 Memory impairment in monkeys following lesions limited to the hippocampus. Behav Neurosci 100:155-160

30. Zola-Morgan S, Squire LR, Amaral DG 1986 Human amnesia and the media temporal region: enduring memory impairment following a bilateral lesion limited to field CA1 of the hippocampus. J Neurosci 6:2950-2967

31. Kim S, Ponka P 1999 Control of transferrin receptor expression via nitric oxidemediated modulation of iron-regulatory protein 2. J Biol Chem 274:33035-33042 